\title{
Development and validation of a simplified method to generate human microglia from pluripotent stem cells
}

Amanda McQuade ${ }^{1,2,3}$, Morgan Coburn 1,2,3, Christina H. Tu 2,3, Jonathan Hasselmann 1,2,3, Hayk Davtyann ${ }^{2,3}$ and Mathew Blurton-Jones ${ }^{1,2,3^{*}}$

\begin{abstract}
Background: Microglia, the principle immune cells of the brain, play important roles in neuronal development, homeostatic function and neurodegenerative disease. Recent genetic studies have further highlighted the importance of microglia in neurodegeneration with the identification of disease risk polymorphisms in many microglial genes. To better understand the role of these genes in microglial biology and disease, we, and others, have developed methods to differentiate microglia from human induced pluripotent stem cells (iPSCs). While the development of these methods has begun to enable important new studies of microglial biology, labs with little prior stem cell experience have sometimes found it challenging to adopt these complex protocols. Therefore, we have now developed a greatly simplified approach to generate large numbers of highly pure human microglia.
\end{abstract}

Results: iPSCs are first differentiated toward a mesodermal, hematopoietic lineage using commercially available media. Highly pure populations of non-adherent $\mathrm{CD}_{4} 3^{+}$hematopoietic progenitors are then simply transferred to media that includes three key cytokines (M-CSF, IL-34, and TGF $\beta-1$ ) that promote differentiation of homeostatic microglia. This updated approach avoids the prior requirement for hypoxic incubation, complex media formulation, FACS sorting, or coculture, thereby significantly simplifying human microglial generation. To confirm that the resulting cells are equivalent to previously developed iPSC-microglia, we performed RNA-sequencing, functional testing, and transplantation studies. Our findings reveal that microglia generated via this simplified method are virtually identical to iPS-microglia produced via our previously published approach. To also determine whether a small molecule activator of TGF $\beta$ signaling (IDE1) can be used to replace recombinant TGF $\beta 1$, further reducing costs, we examined growth kinetics and the transcriptome of cells differentiated with IDE1. These data demonstrate that a microglial cell can indeed be produced using this alternative approach, although transcriptional differences do occur that should be considered.

Conclusion: We anticipate that this new and greatly simplified protocol will enable many interested labs, including those with little prior stem cell or flow cytometry experience, to generate and study human iPS-microglia. By combining this method with other advances such as CRISPR-gene editing and xenotransplantation, the field will continue to improve our understanding of microglial biology and their important roles in human development, homeostasis, and disease.

Keywords: Microglia, Neurodegeneration, GWAS, Stem cells, iPSCs, Hematopoietic precursor cells, Phagocytosis, TGFB, IDE1, Differentiation

\footnotetext{
* Correspondence: mblurton@uci.edu

${ }^{1}$ Department of Neurobiology \& Behavior, University of California, 3014 Gross

Hall, 845 Health Science Rd, Irvine, CA 92697-4545, USA

${ }^{2}$ Sue and Bill Gross Stem Cell Research Center, University of California, 3014

Gross Hall, 845 Health Science Rd, Irvine, CA 92697-4545, USA

Full list of author information is available at the end of the article
}

(c) The Author(s). 2018 Open Access This article is distributed under the terms of the Creative Commons Attribution 4.0 International License (http://creativecommons.org/licenses/by/4.0/), which permits unrestricted use, distribution, and

reproduction in any medium, provided you give appropriate credit to the original author(s) and the source, provide a link to the Creative Commons license, and indicate if changes were made. The Creative Commons Public Domain Dedication waiver (http://creativecommons.org/publicdomain/zero/1.0/) applies to the data made available in this article, unless otherwise stated. 


\section{Background}

Microglia are highly specialized tissue resident macrophages within the brain. Their homeostatic functions include shaping neural circuits through promotion of neuronal growth and differentiation as well as synaptic pruning. Microglia have also been strongly implicated in a number of neurological diseases and injuries. Most recently, genetic studies have identified many genes that are highly expressed in microglia which are associated with altered risk of developing Alzheimer's disease (AD), Parkinson's disease, Frontotemporal Dementia, or Amyolateral Sclerosis [1-4]. These new discoveries have placed microglia and neuroinflammation at the forefront of disease progression emphasizing the need for new model systems to enable the study of human microglia. Yet, microglia have proven to be difficult cells to study given that many differences exist between human and murine microglia [5]. Additionally, there are significant challenges in isolating and culturing these cells $[6,7]$. Primary human microglia can be isolated in relatively limited numbers from postmortem brain tissue or following surgical resection of brain tumors or epileptic foci. However, given the considerable sensitivity of microglia to environmental changes, samples isolated from patients with neurological disease or following the agonal state prior to death, are likely to be activated and may differ depending on disease state, comorbidities, or cause of death. In order to study a more homeostatic human microglia and to utilize modern experimental manipulations such as CRISPR gene editing, many scientists have instead turned to induced pluripotent stem cells (iPSCs).

In the past 3 years, several labs, including our own, have developed various protocols for differentiating microglia-like cells from pluripotent stem cells [8-13]. While the purity, yield, and reproducibility of these different approaches varies considerably, each of these methods produces myeloid cells that exhibit transcriptional profiles and many key functional or morphological characteristics of human microglia. However, the relatively complex nature of these protocols has made it challenging for labs new to stem cell culture or those lacking fluorescence-associated cell-sorting (FACS) core facilities to quickly adopt these approaches. We have therefore developed an appreciably simplified method (iPS-microglia 2.0) to produce both large numbers and highly purified cultures of human microglia. The resulting cells exhibit RNA transcript profiles that are nearly identical to iPS-microglia generated using our previously published protocol [8], but provide a significantly increased yield at a reduced cost and omit the prior need for a hypoxic incubator and FACS capabilities, making the protocol more readily accessible for a wider variety of labs.

\section{Results}

The transcriptome of iPS-microglia 2.0 differentiated without hypoxia or cell sorting are almost identical to those generated using our prior approach

Our new differentiation protocol still mimics in vivo microglia ontogeny by first differentiating iPSCs into hematopoietic progenitor cells (HPCs), followed by passage into microglial differentiation medium, and concludes with a final maturation step by adding neural and astrocytic factors, thereby educating the microglia in a brain-like environment while maintaining a pure, homeostatic population of microglia (Fig. 1).

In order to test whether or not an extensively simplified and commercially available hematopoietic stem cell differentiation protocol could be used to generate equivalent homeostatic microglia, we differentiated four independent iPSC lines, and one ESC line (H9) in parallel using our previously published protocol [8] (iPS-microglia) and the simplified iPS-microglia 2.0 protocol described here. Our prior protocol required FACS to isolate $\mathrm{CD} 43^{+}$hematopoietic progenitors before transitioning to microglial differentiation medium containing IL-34, TGF $\beta-1$, and M-CSF [14-16]. To determine whether FACS sorting was also necessary for our newer approach, we compared three different sorting methods on the same iPSC background. On day 11 when HPCs were ready to be transitioned into microglial medium, we isolated $\mathrm{CD} 43^{+}$HPCs using either FACS, magnetic-activated cell sorting (MACS), or by simply collecting all non-adherent cells. After analyzing transcriptome changes through RNA-sequencing on these samples, we performed unbiased clustering and found that FACS- and MACS-sorted iPS-microglia 2.0 intercluster with unsorted samples demonstrating that these isolation procedures are not necessary for this updated protocol (Additional file 1: Figure S1). Notably, one of the first results we observed with this simplified protocol was that the number of $\mathrm{CD} 43^{+}$HPCs produced using this novel commercially available method was substantially increased, while still maintaining the high degree of purity (90-94\%) for the HPC marker CD43 (Additional file 2: Figure S2). From one million starting iPS cells, 125 million $\mathrm{CD} 43^{+}$cells can be produced, representing an approximately 60 -fold increase over our prior method. Following transition to microglial medium, the four lines of unsorted HPCs were further differentiated and matured. At the final day of microglial maturation, iPS-microglia or iPS-microglia 2.0 were harvested for RNA isolation and analyzed via RNA sequencing.

At a transcriptome level, our new protocol produced homeostatic human microglia that were virtually identical to microglia generated using our prior methods. Principal component analysis of the full transcriptome explained $73 \%$ of variation in all samples within PC1 (44\%) and PC2 (29\%) and revealed that our new 


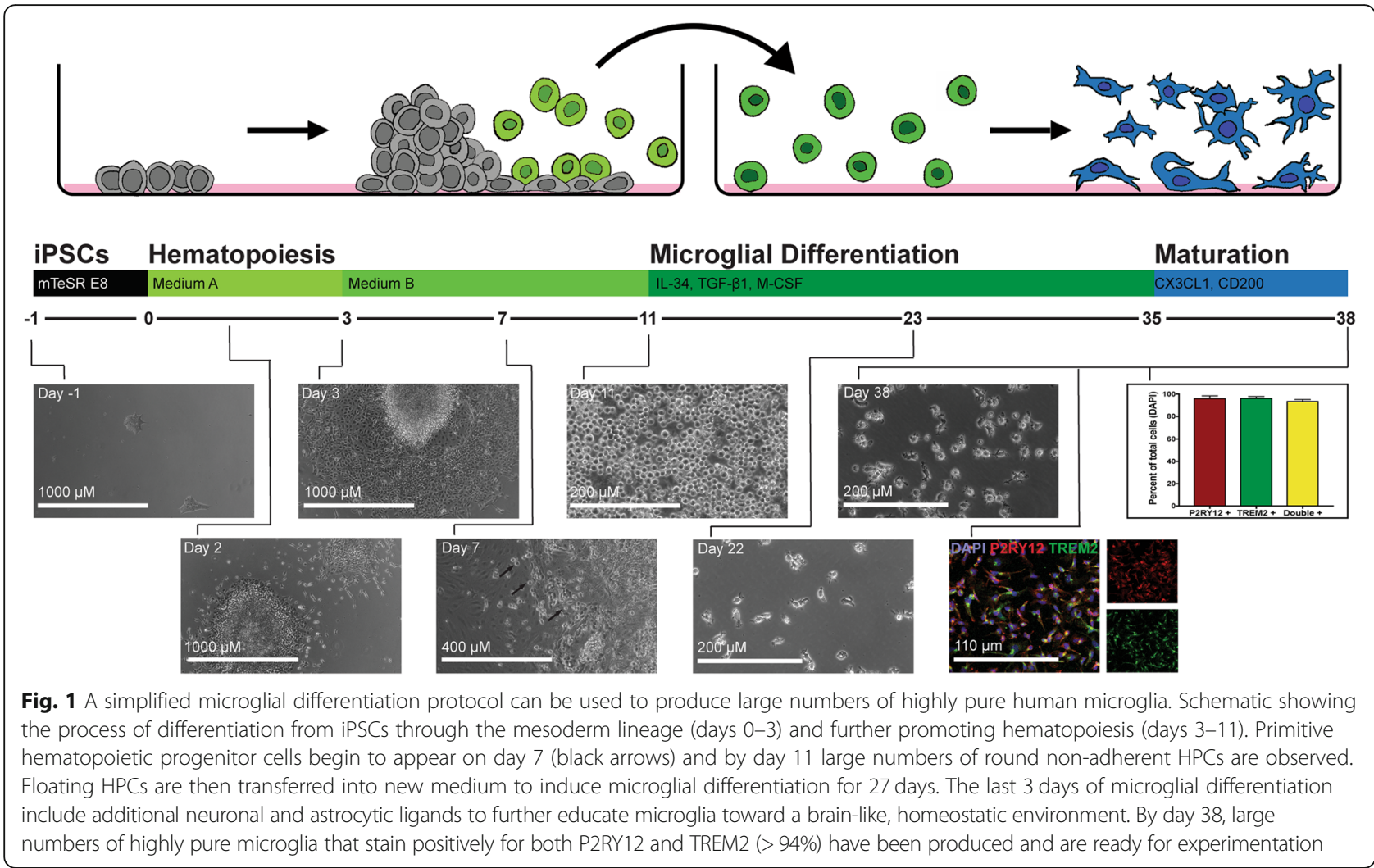

microglia (iPS-microglia 2.0) closely cluster and are interspersed with microglia differentiated using our previously published protocol, yet are highly distinct from human $\mathrm{CD} 4^{+}$or $\mathrm{CD} 14 / \mathrm{CD} 16^{+}$blood monocytes and dendritic cells (Fig. 2a, b, Fig. 3, Additional file 3: Table S1). Consistent with our previous findings, both iPS-microglia and iPS-microglia 2.0 exhibit very similar gene expression profiles to brain-derived cultured human microglia, although some differences between these groups remain. In order to highlight important microglial and monocyte enriched genes, we performed a secondary principal component analysis with a previouslyidentified microglial/monocyte focused gene set which revealed a developmental component (PC1, 48\% of variance) and again shows interclustering of iPS-microglia 2.0 with microglia generated using our previously published approach (Fig. 2b, d). Importantly, this developmental trajectory remains quite distinct from monocytes and dendritic cells (Fig. 2b).

Interestingly, RNA sequencing analysis of HPCs generated using our previously published approach versus HPC 2.0 revealed some intriguing differences between these two populations. Based on the developmental trajectory shown in Fig. 2b, these data suggest that HPC 2.0 samples are closer to microglia on the primary principle component showing the developmental trajectory than
HPCs from our previously described protocol. Indeed HPC 2.0 populations include lower percentages of cells expressing the primitive HPC marker CD235a, although both populations express equivalently high levels of another primitive HPC marker; CD43 which is typically absent in definitive HPCs [17] (Additional file 2: Figure S2, Additional file 4: Table S3). Importantly, although some differences do exist in gene expression between these two HPC populations, very few differences in gene expression persist once HPCs are matured into microglia (Fig. 1, Fig. 2c, d, Additional file 5: Table S2). For example, expression analysis comparing iPS-microglia and iPS-microglia 2.0 revealed only 55 differentially expressed genes. To determine whether these differences occur in a uniform pathway or are indicative of important functional effects that need to be considered, we next used Reactome 2016 gene ontology analysis to examine these 55 genes. This analysis revealed only three significant gene ontology pathways (Platelet activation, signaling and aggregation_Homo sapiens_R-HAS-76002, $\quad \mathrm{FDR}=0.03665 ; \quad$ Chemokine receptors bind chemokines_Homo sapiens_ROHSA-380,108, FDR $=0.03665 ;$ Tryptophan catabolism_Homo sapiens $H S A-72,140, F D R=0.03665$ ). The chemokine receptor pathway is of course important for microglial function, although this pathway was implicated by only 3 differentially expressed genes: CXCL10, CCL5, and PF4. Thus, we 


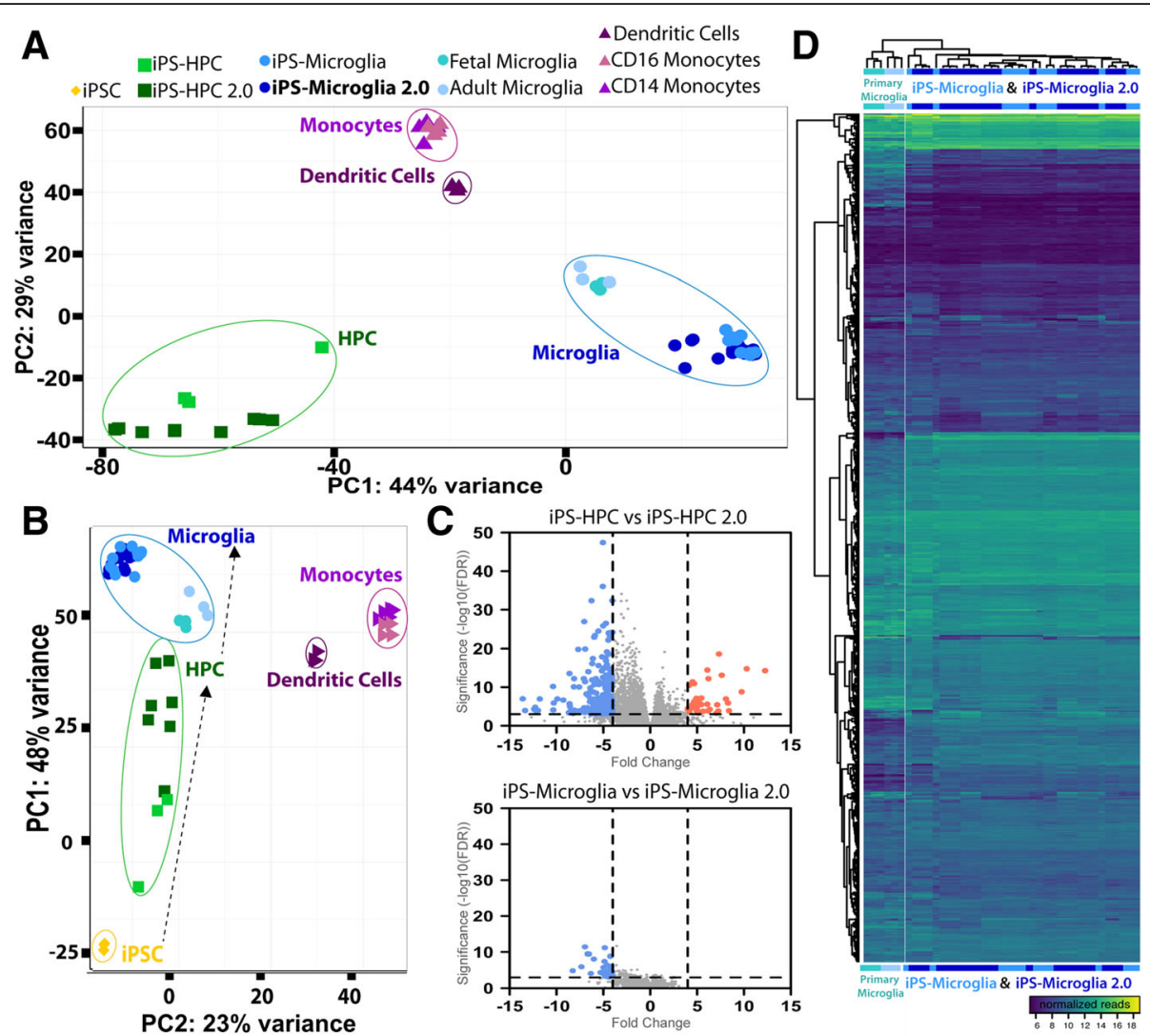

Fig. 2 iPS-microglia 2.0 are virtually identical to iPS-microglia generated using a more complex protocol. a Principle component analysis demonstrates that iPS-microglia 2.0 (dark blue) and iPS-microglia differentiated using our previously published protocol (blue) exhibit highly equivalent gene expression profiles that cluster closely with cultured human fetal and adult microglia (light blue and teal). Additionally, these cells are distinct from human CD14+ monocytes (purple) and CD16+ inflammatory monocytes (pink), and dendritic cells (maroon). b Principal component analysis using a gene list enriched for 882 microglial genes from (Gosselin et al., 2017), further demonstrates the equivalent gene expression between iPS-microglia and fetal and adult microglia. This analysis also highlights the trajectory of differentiation from iPSCs to Microglia and shows the separation between our protocol and monocytic and dendritic cell populations. c Volcano plot of differential expression analysis $(p<0.001, \log 2(\mathrm{FC})>2$ ) between iPS-HPC and iPS-HPC 2.0 samples (top) as well as iPS-microglia and iPS-microglia 2.0 (bottom). Significantly increased or decreased genes are shown in coral or blue respectively. $\mathbf{d}$ Heatmap using 882 microglial-enriched genes further demonstrates the highly similar gene expression profiles between iPS-microglia and iPS-microglia 2.0 and the close similarity of both cell populations to fetal and adult cultured microglia

expect that microglial functional activity will be largely equivalent between iPS-microglia and iPS-microglia 2.0.

\section{Functional validation of iPS-microglia 2.0}

To determine whether the functional activity of iPSmicroglia 2.0 is indeed equivalent to microglia generated using our prior approach, we next compared phagocytic activity of cells generated using both methods [8]. Since the ability of microglia to clear pathogens and extracellular aggregates via phagocytosis is an important aspect of microglial function, we exposed microglia to several different substrates and measured the percentage of cells which phagocytose each substrate using an Amnis Imagestream which combines flow cytometry and high throughput immunofluorescence (Fig. 4). As expected, the levels of phagocytosis differed between the three varying substrates with beta-amyloid fibrils producing the highest response. S. aureus bioparticles, a TLR 1,2,6 agonist produced an intermediate degree of phagocytosis and $\mathrm{Zy}$ mosan, a TLR 2/Dectin 1 agonist from S. cerevisia induced the lowest level of phagocytosis (Fig. 4). Importantly, regardless of the differential response to these three phagocytic substrates, iPS-microglia and iPS-microglia 2.0 exhibited identical rates of phagocytosis for each of the substrates, demonstrating that this simplified differentiation method does not alter this important microglial function (Fig. 4).

\section{iPS microglia 2.0 engraft well into xenotransplantation- compatible MITRG mice}

We previously demonstrated that iPS-microglia can engraft and ramify, fulfilling characteristic microglia morphology 

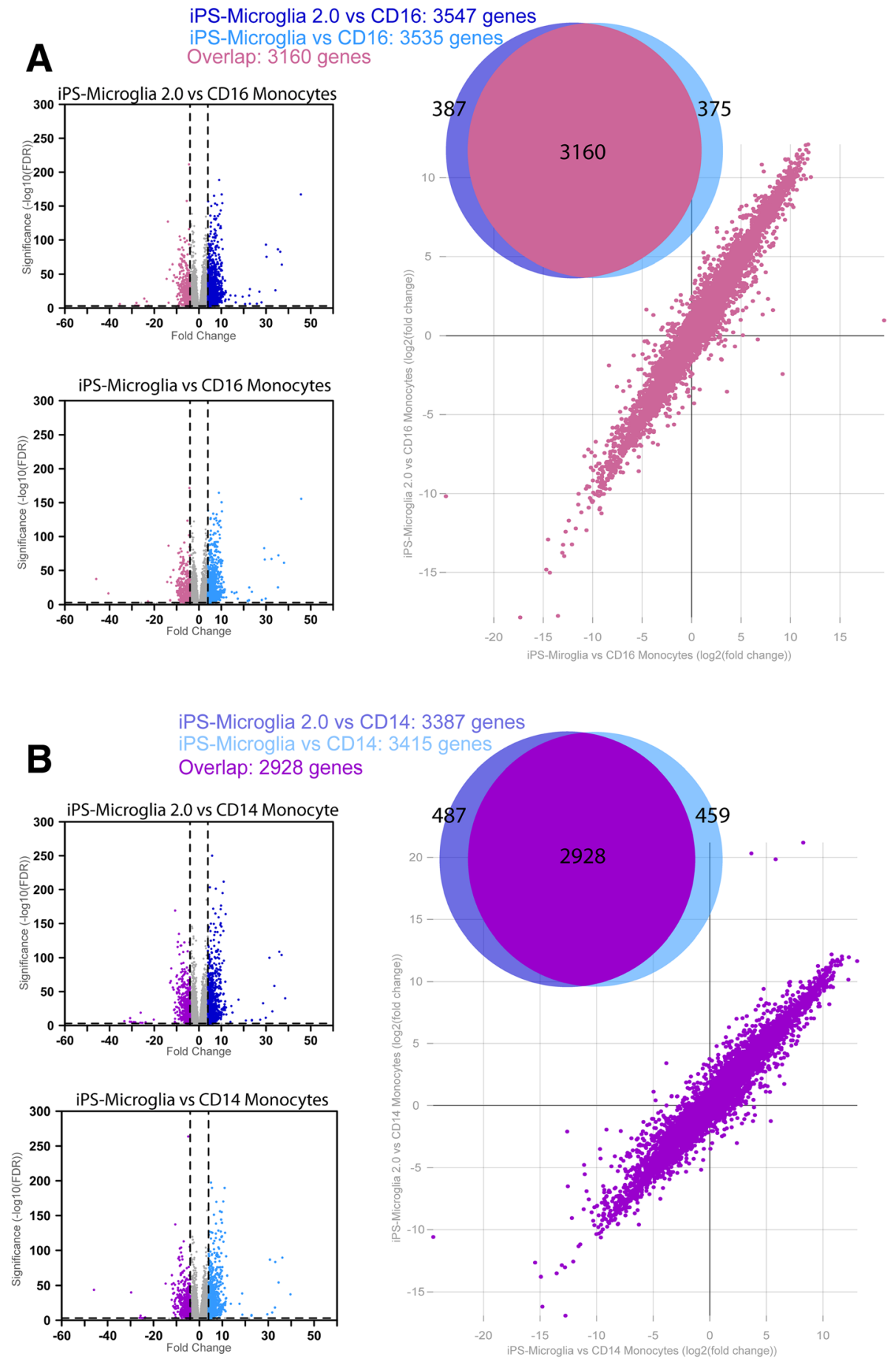

Fig. 3 iPS-microglia 2.0 are distinct from $\mathrm{CD}_{1} 4^{+}$and $\mathrm{CD} 16^{+}$monocytes Microglia differentiated using our published protocol are distinct from CD $14^{+}$monocytes and CD16 ${ }^{+}$inflammatory monocytes. In order to ensure our iPS-microglia 2.0 are similarly distinct from monocytes, differential expression analysis was computed with DEseq2. a Volcano plots of differentially expressed genes comparing genes enriched in CD $16^{+}$monocytes (pink) with genes enriched in iPSmicroglia 2.0 (dark blue) or iPS-microglia (light blue) show many significant differences between monocytes and microglia. Venn diagrams and comparative fold change plots of differentially expressed genes show that the vast majority of differences are identical between iPS-microglia and iPS-microglia 2.0 when compared to CD16 ${ }^{+}$monocytes. Direct comparisons of the fold change expression level (TPM) of every gene are shown in comparative fold change plots which demonstrate the striking similarity of differential expression when iPS-microglia and iPS-microglia 2.0 are each compared to $\mathrm{CD}_{16}{ }^{+}$monocytes. $\mathbf{b}$ The same is true for comparisons of iPS-microglia and iPS-microglia 2.0 with CD14 ${ }^{+}$ monocytes (purple) 


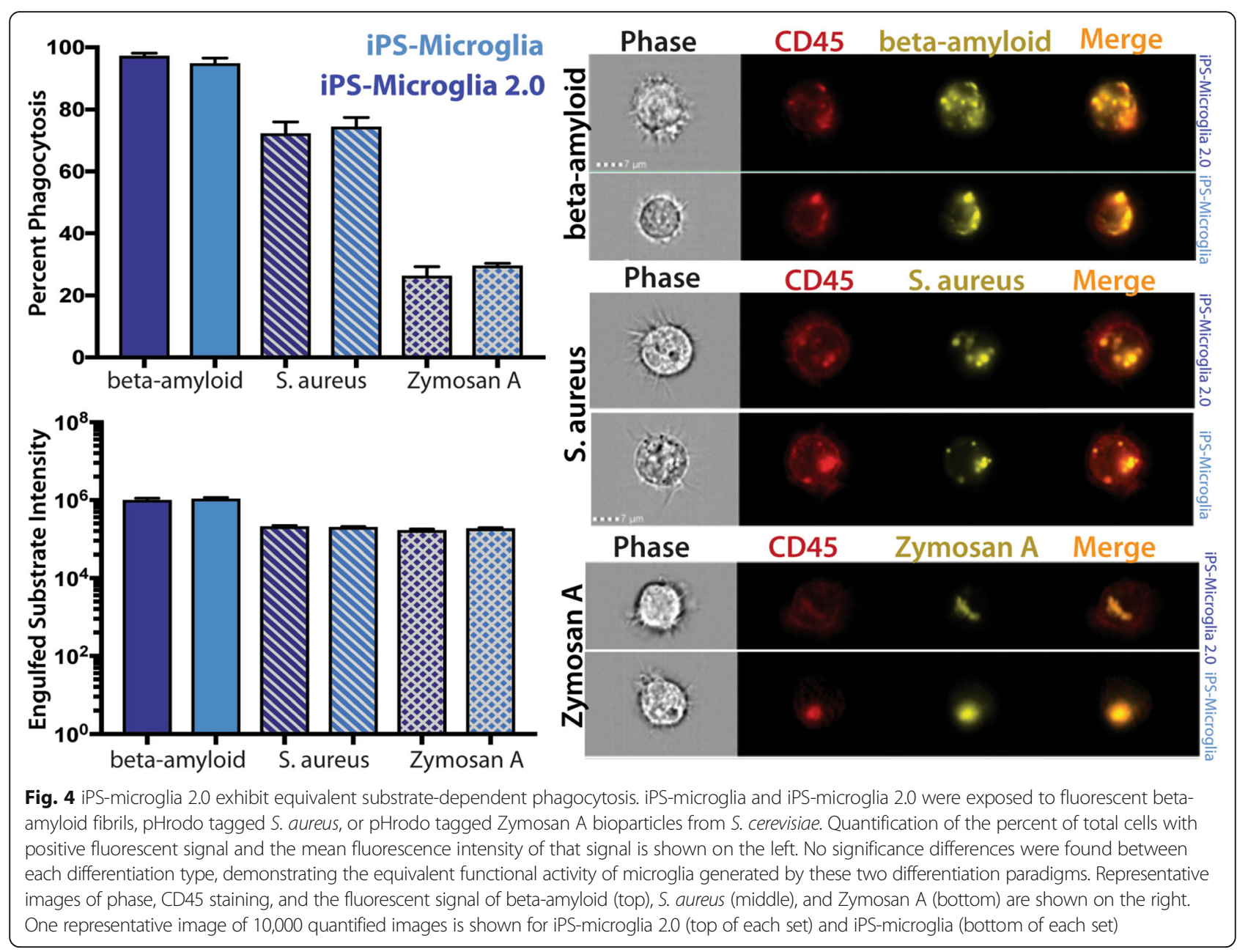

and marker expression in the brains of xenotransplantation-compatible MITRG (Knock-out: Rag2; Il2rg; Knock-in: M-CSFh; IL-3/GM-CSFh; TPOh) mice [8]. Thus, we aimed to further validate the identity of our iPS-microglia 2.0 through intracranial transplantation of iPS-microglia 2.0 into MITRG mice, and to compare this engraftment to equivalently transplanted iPS-microglia that were generated using our previously described differentiation method. In each case, fully mature microglia were transplanted into the hippocampus and overlaying cortex of adult mice which were sacrificed after 2 months for histological examination of morphology and key marker expression. Both iPS-microglia and iPS-microglia 2.0 can be identified within the mouse brain via expression of the human-specific nuclear marker, Ku80 (Fig. 5, green). Importantly, regardless of the differentiation method, transplanted human microglia display typical microglial morphology, extending complex branching processes. Both iPS-microglia and iPS-microglia 2.0 also express the microglial/monocyte marker Iba1 (Fig. 5, Overlay images C, G, K, \& O, red) and the homeostatic microglial marker P2RY12 (Fig. 5 Overlay images, D, H, L, \& P, red) in both cortex and hippocampus, indicating that these cells engraft well and remain homeostatic. Transplanted iPS-microglia 2.0 also exhibit the tiling and distinct niches typical of in vivo microglia, and can be seen interspersed with the endogenous population of mouse microglia (Fig. 5, arrows indicate $\mathrm{Iba1}^{+} / \mathrm{Ku} 80^{-}$ mouse cells). Taken together, these findings further demonstrate that iPS-microglia 2.0 are equivalent to microglia generated using our previously published protocol and can be readily transplanted into MITRG mice to enable in vivo studies of human microglia.

\section{Small molecule activation of TGF $\beta$ signaling produces} microglia-like cells that are similar, but transcriptionally distinct from iPS-microglia 2.0

TGF $\beta 1$ is a crucial astrocyte-derived cytokine that promotes microglial homeostasis $[15,18]$. TGF $\beta 1$ signaling results in phosphorylation of $\operatorname{smad} 2 / 3$ and ultimately up-regulates expression of CX3CR1, an important receptor for microglial function and survival $[19,20]$. Indeed, removal of either TGF $\beta 1$ or CX3CR1 greatly decreases microglia populations in murine models $[15,20]$. As we have previously shown, removal of TGF $\beta 1$ from iPS-microglia 

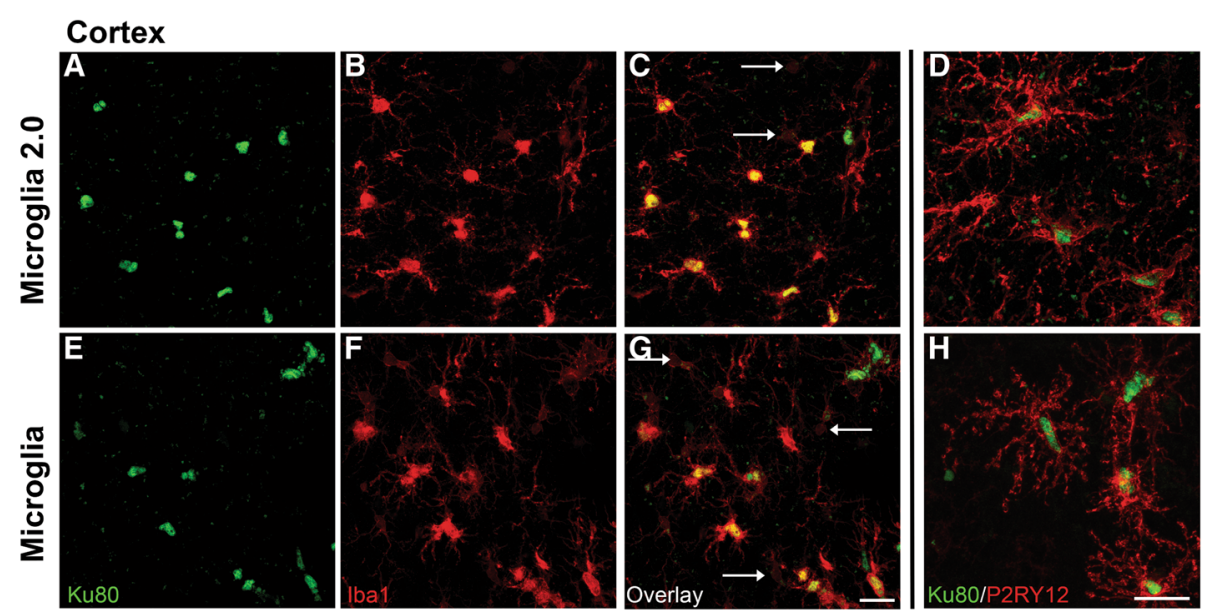

\section{Hippocampus}
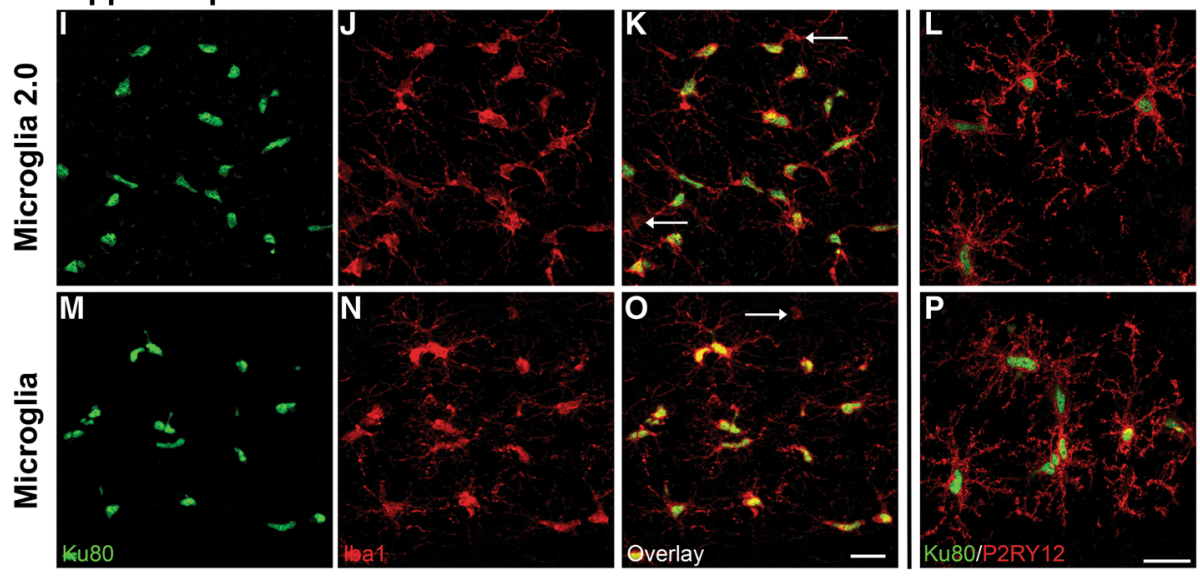

Fig. 5 Transplanted iPS-microglia 2.0 display typical microglial markers and morphology comparable to our previously described iPS-microglia. Adult 2 month old MITRG mice were transplanted with (a-d \& $\mathbf{i}-\mathbf{j}$, top rows) iPS-microglia 2.0 or (e-h \& $\mathbf{m}-\mathbf{p}$, bottom rows) iPS-microglia. Brains were harvested 2 months post-transplant. Representative images of cortical (a-h) and hippocampal (i-p) transplanted cells demonstrate complex process ramification and typical tiling. Transplanted iPS-microglia and iPS-microglia 2.0 both express Iba-1 (Overlay images $\mathbf{c}, \mathbf{g}, \mathbf{k}, \& \mathbf{0}$, red) and the microglia specific marker, P2RY12 (Overlay images $\mathbf{d}, \mathbf{h}, \mathbf{l}, \& \mathbf{p}$, red) and demonstrate human nuclear staining (Ku80, green). Additionally, transplanted human microglia can be seen integrating and tiling with the endogenous mouse microglia population (Arrows indicate Iba1+/Ku80- cells)

for even $24 \mathrm{~h}$ also results in dramatic changes in the microglial transcriptome, including down-regulation of homeostatic signatures [8].

In order to increase cost-efficiency during iPS-microglia 2.0 differentiation, we attempted to replace recombinant TGF $\beta 1$ with Inducer of Definitive Endoderm 1 or 2 (IDE1, IDE2). As its name suggests, IDE has been used to differentiate iPS cells into definitive endoderm through activation of TGF $\beta$ signaling [21]. More specifically, IDE1/2 have been shown to induce phosphorylation of the downstream TGF $\beta$ signaling molecule smad2 [21]. This led us to hypothesize that IDE1 or IDE2 could induce expression of microglial genes in an equivalent fashion to recombinant TGF $\beta$ if added after mesoderm formation and hematopoiesis.

To determine whether IDE1 or IDE2 could replace recombinant TGF $\beta$ in our differentiation protocol, HPCs harvested on day 10 were placed into microglia differentiation media and varying concentrations of IDE1 or IDE2 $(1 \mu \mathrm{M}, 10 \mu \mathrm{M}, 100 \mu \mathrm{M}, 1000 \mu \mathrm{M})$ were added in place of TGF $\beta$ (Fig. 6a). During the first 4 days of microglia differentiation we used an Incucyte live imaging system to examine the growth kinetics of each group to provide an initial assessment of the effects of varying IDE concentrations. Surprisingly, this analysis demonstrated that IDE2, regardless of concentration, impaired normal microglial proliferation and thus was not studied further. In contrast, IDE1 was able to mimic the typical growth kinetics observed in control cells differentiated in parallel and maintained in normal TGF $\beta$-containing medium. Because the control TGF $\beta$ microglial growth curve fell between the $10 \mu \mathrm{M}$ and $1 \mu \mathrm{M}$ IDE1 curves, we next adjusted the IDE1 concentrations to include a $5 \mu \mathrm{M}$ dose. In addition, two higher concentrations of IDE1 

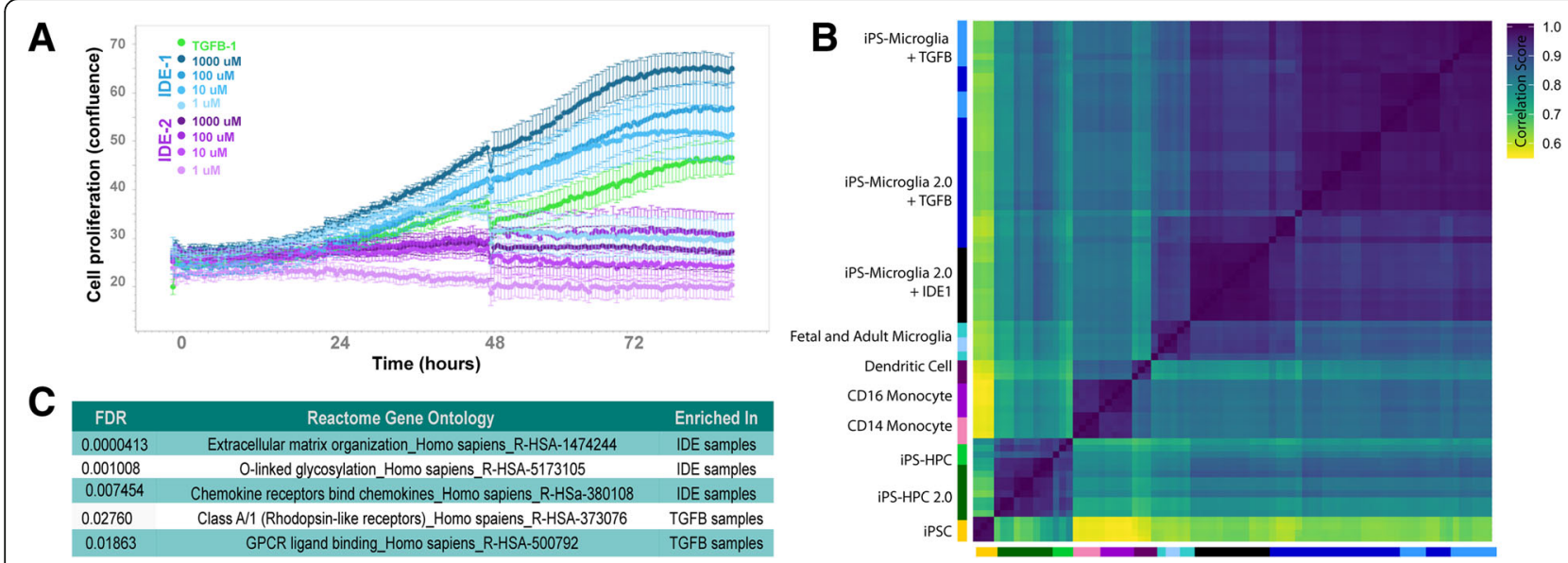

Fig. 6 The small molecule compound IDE1 can be used in place of TGF $\beta-1$ to produce iPS-microglia. IDE1 and IDE2 were added to microglia cultures in place of TGF $\beta-1$ at the indicated concentrations. a Growth curves from the first 3.5 days of microglial differentiation show that IDE2 is insufficient to allow proliferation of these cells. In contrast, IDE1 (blue) at lower concentrations shows similar growth kinetics to TGF $\beta$ control cells (green). b Correlation matrix displaying all samples analyzed in this manuscript shows cells differentiated with IDE1 cluster closely with iPS-microglia 2.0 and are actually more similar to fetal and adult microglia than TGF $\beta$ control microglia. c Gene ontology analysis using the Reactome database displays differences between IDE1 treated cells and TGF $\beta$ (FDR $<0.001, F C>2$ ). Enrichment in IDE samples reflects an increased expression of genes within this GO family in IDE1 treated cells

$(50 \mu \mathrm{M}$, and $500 \mu \mathrm{M})$ were also included as the growth kinetic measurements suggested that these concentrations might further increase the yield of microglia.

All groups were differentiated in parallel for the complete 38 day paradigm before RNA-sequencing was performed. To compare these new samples with our iPS-microglia, fetal and adult microglia, and other cell types, we generated a correlation matrix (Fig. 6b) which demonstrated that IDE1 iPS-microglia remain distinct from monocytes and dendritic cells and cluster closely with our other iPS-microglia. IDE-treated cells also exhibited strong expression of key microglial genes including CSF1R, P2YR12, TREM2, OLFML3, HEXB, and C1Q (Additional file 6: Table S4). Based on the hierarchical clustering within our correlation matrix (Fig. 6b), we find that microglia differentiated in IDE1 have transcriptomic profiles that are more similar to primary cultured microglia (fetal and adult microglia). One possible explanation for this finding is that IDE1 may have greater stability within the culture media than TGF $\beta 1$ and thus provide a more uniform and consistent activation of TGF $\beta$ signaling pathways. Alternatively, IDE1 could potentially provide increased specificity by only targeting smad2 signaling. To further understand the comparative effects of IDE1 versus TGF $\beta 1$ we further compared these groups of iPS-microglia using DEseq2 to highlight any differentially expressed genes (Additional file 6: Table S4). Interestingly, gene ontology analysis of these differentially expressed genes revealed only five pathways that were significantly enriched between these two populations of microglia (Fig. 6c), again showing their strong similarity. Taken together these data suggest that IDE1 can indeed be used in place of TGF $\beta 1$, although researchers should also consider these differentially altered pathways and genes in there decision whether or not to use this further modified approach.

\section{Discussion}

In recent years, the importance of microglia in brain development, homeostasis, and disease has become increasingly clear. Because microglia have been implicated in many neurological diseases and injuries including neurodegeneration, traumatic brain injury, and developmental disorders, several groups have developed methods to try to make these cells more accessible for neurological research. Until recently, microglia could only be studied through brain biopsies, postmortem analysis, or in animal models. Although mouse models of neuroinflammation have been extremely useful in uncovering important new findings, many differences exist between human and murine microglia. For example, one recent study identified several co-regulated myeloid gene expression modules that occur in human $\mathrm{AD}$, but do not occur in AD mouse models [22]. Likewise, many differences exist between the murine and human complement system that is closely linked to neurodegenerative diseases including $\mathrm{AD}$ [6]. At least two microglial-expressed $\mathrm{AD}$ risk genes, CR1 and MS4A4A, have no murine ortholog, further highlighting the challenges of studying the role of microglia in human disease using mouse models alone.

To study human microglia, some highly skilled groups have turned to human biopsy material. Working closely with neurosurgeons, these researchers have developed methods to isolate human microglia from brain tissue removed during a surgical resection of a brain tumor or 
intractable epileptic foci $[5,7,23]$. Using this approach, researchers have uncovered exciting data and greatly advanced our understanding of the human microglial transcriptome. However, epileptic foci and tumor tissue induce neuroinflammation and despite best efforts to avoid isolating microglia from 'diseased-effected tissue' it is likely that microglia isolated from these patients exhibit considerable variation and alterations in activation state $[24,25]$.

Another strategy for studying human microglia involves the isolation of microglia or their nuclei from postmortem brain tissue. Using this approach, researchers have uncovered important age-related differences in the human microglial transcriptome [26]. Still, it remains unclear whether the agonal state that precedes death, inflammatory co-morbidities, or post-mortem delay might influence microglial gene expression. In the case of Alzheimer's disease, most patients die from an accompanying infectious disease such as Pneumonia [27, 28]. Interestingly, animal models of Pneumonia exhibit significant changes in brain microglial activation state $[29,30]$. Thus, it is likely that this and other common infectious co-morbidities can complicate the interpretation and analysis of postmortemisolated human microglia.

Given the considerable challenges with isolation and study of postmortem or biopsied human microglia, several groups, including our own, developed protocols which utilize the power of stem cells to produce human microglia in vitro [8]-[13]. These methods have begun to enable more detailed mechanistic studies of human microglia by allowing controlled experimental treatments, drug testing, and genetic manipulation. However, the currently existing protocols are relatively complicated and can be challenging to adopt, especially for groups with little prior stem cell experience. Thus, to address this challenge we developed and validated the greatly simplified and refined method presented here. In comparing this new method to our previously published differentiation protocol, we confirm that iPS-microglia 2.0 show highly similar RNA transcript profiles to iPS-microglia as well as primary fetal and adult microglia. In addition, iPS-microglia 2.0 remain distinct from blood monocytes and importantly display largely the same differentially expressed genes between microglia and monocytes as our previously published iPS-microglia.

To further investigate and characterize iPS-microglia 2.0 we functionally validated these cells by examining phagocytosis of three different substrates; Staphylococcus aureus, Zymosan A, and fibrillar beta-amyloid. While each substrate exhibited differential degrees of phagocytosis, these levels were equivalent between our previously described iPS-microglia and iPS-microglia 2.0. Lastly, to determine whether iPS-microglia 2.0 can also be used for in vivo studies, we transplanted microglia derived via both methods into xenotransplantation-compatible MITRG mice, confirming that engraftment, in vivo morphology, and marker expression was equivalent between iPS-microglia and iPS-microglia 2.0. Taken together, these functional and in vivo experiments further support the conclusion that microglia generated via these two methods are virtually identical.

In addition, we tested IDE1 as a small molecule agonist of TGF $\beta$ signaling cascades. To this end, we confirmed that substitution of TGF $\beta 1$ with IDE1 produced cells that are similar to iPS-microglia 2.0, and additionally highly similar to adult and fetal primary microglia. We have provided differential gene expression analysis to highlight the important differences between IDE- and TGF $\beta 1$-treated iPS-microglia 2.0, which researchers should consider when deciding whether to use TGF $\beta$ or cost-saving IDE1 for iPS-microglia generation.

\section{Conclusions}

In summary, we provide detailed methods and validation of a greatly simplified protocol to produce significantly increased numbers of pure human microglia. The RNA-sequencing, functional validation, and transplantation studies presented here clearly demonstrate that highly pure populations of human iPS-microglia can be generated via this greatly simplified protocol. We anticipate that this streamlined and highly reproducible protocol will enable many more interested researchers to now study human microglia, leading to further breakthroughs in this field.

\section{Methods}

Ethics Statement: All experiments were carried out according to human stem cell (hSCRO) and animal use (IACUC) protocols that were approved by the University of California, Irvine.

Find the complete catalog of materials and catalog numbers in Additional file 7: Table S5.

\section{Simplified differentiation of iPSCs to HPCs}

Improved and simplified differentiation of iPSCs to $\mathrm{CD} 43^{+}$primitive hematopoietic progenitor cells (HPCs) is achieved using Stem Cell Technologies STEMdiff ${ }^{\text {mi }}$ Hematopoietic Kit (Catalog \# 05310). On day - 1, feeder-free iPSCs that have been expanded in TeSR-E8 media are passaged with ReLeaSR (STEMCELL technologies) into mTeSR E8 medium with $0.5 \mu \mathrm{M}$ Thiazovivin onto matrigel coated $(1 \mathrm{mg} / \mathrm{mL})$ 6-well plates (Corning Costar). Small aggregates of $\sim 100$ cells each are plated at $10-20$ aggregates per $\mathrm{cm}^{2}$. The initial plating density is critical as higher density impairs mesoderm differentiation and lower density decreases yield. Thus one can plate iPSCs at 2-3 different densities and 
select the wells on day 0 that have optimal density to proceed with. When approximately two 100 cell colonies per $\mathrm{cm}^{2}$ have been achieved, replace TeSR-E8 medium with medium A (Basal medium plus Supplement A at 1:200 dilution, $2 \mathrm{~mL}$ per well of a 6 -well). On day 2 (48 h after original media change), do not fully change media, but rather replace $50 \%$ medium A, $1 \mathrm{~mL}$ per well of a 6-well. On day 3 , carefully remove all media by tilting the plate to one side and aspirating from the edge. Then add 2 $\mathrm{mL} /$ well medium B (Basal medium plus supplement B at 1:200). Without removing media, supplement with $1 \mathrm{~mL} /$ well of medium B on days $5,7,9$. On day 10 and again on day and 12, non-adherent cells may be collected. To maintain purity, do not wash cells off the well, but merely remove medium with non-adherent cells carefully and centrifuge 300 x G 5 min. After centrifugation, replace conditioned medium back to each well and add $1 \mathrm{~mL}$ fresh medium B if further collection on day 12 will be completed.

FACS analysis has confirmed that these non-adherent cells represent highly pure populations (>93\%) of $\mathrm{CD} 3^{+}$hematopoietic progenitor cells (Additional file 2: Figure S2). Importantly, simply collecting the floating cells is all that is required to isolate large numbers of highly purified $\mathrm{CD} 43^{+}$cells. No FACS or MACS isolation is required as identical microglia are produced using any of these three methods (Additional file 1: Figure S1). However, because the cells are not being sorted for purity, the collection of non-adherent cells must be carefully completed. Do not spray medium over adherent cells to wash as this will loosen cells which are not $\mathrm{CD} 43^{+}$and decrease culture purity.

At this point, HPCs may be frozen at 2-4 million cells per $\mathrm{mL}$ in BamBanker (Wako). If frozen, HPCs should be thawed directly into microglial differentiation medium with cytokines (below) and plated onto Matrigel-coated plates at 10,000 cells per $\mathrm{cm}^{2}$. We typically find that viability post-thaw is between $70-95 \%$, with improved viability when greater densities of HPCs are thawed together.

\section{Updated differentiation of $\mathrm{CD}^{+} 3^{+} \mathrm{HPCs}$ to iPS-microglia 2.0}

Volumes specified for $35 \mathrm{~mm}$ well (1 well of a 6-well plate).

On day 0 of iPS-microglia differentiation, plate HPCs at 10,000 cells per $\mathrm{cm}^{2}$ onto $1 \mathrm{mg} / \mathrm{mL}$ Matrigel-coated plates $(100,000$ per $35 \mathrm{~mm}$ well). Plate cells into iPS-microglia medium at $2 \mathrm{~mL}$ per $35 \mathrm{~mm}$ well: DMEM/ F12, 2X insulin-transferrin-selenite, 2X B27, 0.5X N2, $1 \mathrm{X}$ glutamax, $1 \mathrm{X}$ non-essential amino acids, $400 \mu \mathrm{M}$ monothioglycerol, $5 \mu \mathrm{g} / \mathrm{mL}$ insulin. Immediately before use, microglial medium should be supplemented with $100 \mathrm{ng} / \mathrm{mL}$ IL-34, $50 \mathrm{ng} / \mathrm{mL}$ TGF $\beta 1$, and $25 \mathrm{ng} / \mathrm{mL}$ M-CSF (Peprotech) taken from single-use frozen aliquots (important: do not freeze/thaw these cytokines as it will significantly impair differentiation and yield as well as induce activation. It is crucial to thaw cytokines immediately before use). Throughout the differentiation of HPCs to microglia, these cells will predominantly grow non-adherently. On days 2, 4, 6, 8, and 10, add 1 $\mathrm{mL}$ fresh media plus freshly thawed tri-cytokine cocktail. Cytokines are diluted to the concentrations listed above before adding to conditioned medium. Do not fully remove media during the microglial differentiation as the cells secrete paracrine cytokine signals and will not properly differentiate upon removal of those. On day 12 , collect $6 \mathrm{~mL}$ media from each $35 \mathrm{~mm}$ well leaving $1 \mathrm{~mL}$ conditioned medium on the plate. Centrifuge non-adherent cells in removed medium for $5 \mathrm{~min}$ at $300 \mathrm{x}$ G. Aspirate medium and resuspend non-adherent cells in 1 $\mathrm{mL}$ fresh medium plus tri-cytokine cocktail per $35 \mathrm{~mm}$ well and add back to the same well which contains the $1 \mathrm{~mL}$ conditioned medium. Continue to supplement media $(1 \mathrm{~mL})$ on days $14,16,18,20,22$, and 24 . On day 25 , centrifuge cells leaving $1 \mathrm{~mL}$ conditioned media per $35 \mathrm{~mm}$ well as on day 12 . On day 25 , cells should be resuspended in microglia media plus $100 \mathrm{ng} / \mathrm{mL}$ IL-34, 50 $\mathrm{ng} / \mathrm{mL}$ TGF $\beta 1,25 \mathrm{ng} / \mathrm{mL}$ M-CSF, $100 \mathrm{ng} / \mathrm{mL}$ CD200 and $100 \mathrm{ng} / \mathrm{mL}$ CX3CL1 to further mature microglia and ensure homeostasis. On day 27, feed cells with microglia media with five cytokine cocktail ( $1 \mathrm{~mL}$ per well). On day 28 cells collected for RNA sequencing or use for transplantation or functional assays. If necessary, cells can be maintained for 1-2 additional weeks via media supplementation as above, although longer-term culture is not advised.

\section{Isolating RNA}

Total RNA was isolated from cells using RNeasy Mini kit (Qiagen). Approximately 1 million iPS-microglia cells were lysed in RLT buffer and RNA was isolated per manufacturer's instructions with DNAse treatment (10 $\mathrm{min})$ and increased spin times to maximize yield $(16,000$ $\mathrm{x} G$ for $1.5 \mathrm{~min}$ ). RNA integrity was measured using the Bioanalyzer Agilent 2100. All libraries were prepared from samples with RNA integrity values $\geq 9.7 .500 \mathrm{ng}$ RNA per sample was used to create RNA-seq libraries through the Illumina TruSeq mRNA stranded protocol. Each sample was then sequenced in the Illumina HiSeq 4000 platform.

\section{RNA sequencing analysis}

RNA sequencing read integrity was verified using FastQC. BBDuk was used to trim adapters and filter out poor quality reads [31]. Reads were aligned to the GRCh.38.12 human transcriptome using Kallisto [32]. Lowly expressed genes (expression count summed over all samples $<10$ ) were removed before differential expression analysis. Differential Expression of TPM was calculated using DESeq2 [33]. An FDR cutoff of 0.001 
and fold change of at least 2 was used to determine differentially expressed genes (Additional file 3: Table S1, Additional file 4: Table S2, Additional file 5: Table S3, Additional file 6: Table S4). Visualizations were constructed in part using $R$ in addition to the Genialis visual informatics platform (app.genialis.com) [34]. Gene ontology analysis was performed using EnrichR.

\section{Phagocytosis assay}

Phagocytic activity of iPS-microglia was examined using the the Amnis Imagestream (Millipore) to combine immunofluorescence and flow cytometry. iPS-microglia or iPS-microglia 2.0 were treated with either $1 \mu \mathrm{g} / \mathrm{mL}$ pHrodo tagged zymosan A beads, $20 \mu \mathrm{g} / \mathrm{mL}$ S. aureus, or $2 \mu \mathrm{g} / \mathrm{mL}$ fluorescent beta-amyloid (Anaspec). After allowing $1 \mathrm{~h}$ at 37 degrees for phagocytosis, microglia were resuspended in cold FACS buffer (DPBS, 1\% BSA, $0.5 \mathrm{mM}$ EDTA) and stained for $30 \mathrm{~min}$ at 4 degrees with 1:100 anti-CD45 (Biolegend, clone HI30) and Zombieviolet live/dead stain. 10,000 events were captured for each sample which were gated for in focus, live cells before analysis. IDEAS software was used to generate masks of internalized signal (substrate within CD45) and percent of cells with internalized substrates were calculated as well as mean fluorescent intensity which remained constant for each cell type.

\section{Animals}

All animal procedures were conducted in accordance with the guidelines set forth by the National Institutes of Health and the University of California, Irvine Institutional Animal Care and Use Committee. The MITRG mouse was purchased from Jackson Laboratories (stock \#017711); briefly, this strain was developed on a BALB/c background containing two knockouts alleles: Rag2$\left(\operatorname{Rag}^{\text {tm1.1Flv }^{-1}}\right)$ and il2 $\mathrm{\gamma c}^{-}\left(\mathrm{Il} 2 \mathrm{rg}^{\mathrm{tm} 1.1 \mathrm{Flv}}\right)$; and three humanized knock-in alleles: hCSF-1 (Csf1 $\left.{ }^{\text {tm1(CSF1)Flv }}\right)$, h-IL-3/GM-CSF $\left(\mathrm{Csf} 2 / \mathrm{Il} 3^{\text {tm1.1(CSF2,IL3)Flv }}\right)$, and hTPO (Thpo $\left.{ }^{\text {tm1.1(TPO)Flv }}\right)$. All mice were age and sex matched and group housed on a 12 $\mathrm{h} / 12 \mathrm{~h}$ light/dark cycle with food and water ad libitum.

\section{Adult intracranial transplants}

All mouse surgeries and use were performed in strict accordance with approved $\mathrm{NIH}$ and AALAC-certified institutional guidelines. Direct intracranial injections of iPS-microglia into the cortex and hippocampus were performed on adult MITRG mice. Briefly, adult mice (2-3 months old) were anesthetized under continuous isoflurane and secured to a stereotaxic frame (Kopf). Using a 30-guage needle affixed to a $10 \mu \mathrm{L}$ Hamilton syringe, mice received $2 \mu \mathrm{L}$ of mature iPS-microglia suspended in sterile $1 \mathrm{X}$ DPBS at 50,000 cells $/ \mu \mathrm{L}$ at each injection site. Transplantation was conducted bilaterally in the cortex and hippocampus at the following coordinates relative to bregma: anteroposterior, $-2.06 \mathrm{~mm}$; dorsoventral, $-1.75 \mathrm{~mm}$ (hippocampus), $-0.95 \mathrm{~mm}$; mediolateral, $\pm 1.75 \mathrm{~mm}$. Cells were injected at a rate of 50,000 / 30 s with $4 \mathrm{~min}$ in between injections. The needle was cleaned with consecutive washes of PBS, $70 \%$ ( $\mathrm{vol} / \mathrm{vol}$ ) ethanol, and PBS in between hemispheres and animals. Animals were allowed to recover on heating pads before being placed in their home cages and received $2 \mathrm{mg} / \mathrm{mL}$ Acetaminophen (Mapap) diluted in water for five days. Animals were perfused 2 months following surgery with 1X PBS followed by $4 \%$ paraformaldehyde, entire brains were removed for immunohistochemistry and confocal microscopy.

\section{Immunohistochemistry and confocal microscopy}

Fixed half brains were first cryoprotected in a 30\% sucrose and $0.05 \% \mathrm{NaN}_{3}$ solution in $1 \mathrm{X}$ PBS for $72 \mathrm{~h}$. Tissue was then sectioned into $40 \mu \mathrm{m}$ thick slices on a freezing microtome (Leica SM 2010R), and stored in $0.05 \% \mathrm{NaN}_{3}$ solution in $1 \mathrm{X}$ PBS as free floating wells. For staining, tissue was blocked for $1 \mathrm{~h}$ in $1 \mathrm{X}$ PBS, $0.2 \%$ Triton X-100, and $10 \%$ goat serum. Immediately following blocking, sections were placed in primary antibodies diluted in $1 \mathrm{X}$ PBS and $1 \%$ goat serum and incubated overnight on a shaker at $4{ }^{\circ} \mathrm{C}$. Sections were labeled with combinations of anti-Ku80 (1:250; Abcam ab79220), anti-Iba1 (1:200; Wako 019-19,741), anti-P2RY12 (1:200; Sigma HPA014518) and mounted with DAPI Fluoromount (SouthernBiotech). Immunofluorescent sections were then visualized and captured using an Olympus FX1200 confocal microscope. Images represent confocal Z-stack taken with equivalent laser and detection settings.

\section{Additional files}

\begin{abstract}
Additional file 1: Figure S1. FACS and MACS sorted HPCs differentiate into equivalent microglia as unsorted HPCs. Hierarchical clustering of all iPSmicroglia 2.0 samples show that MACS (fuchsia) and FACS (purple) sorting at the HPC stage has no effect on the final differentiated microglia as these samples intercluster with iPS-microglia 2.0 produced from floating unsorted HPCs (blue). (TIF $11571 \mathrm{~kb}$ )

Additional file 2: Figure S2. Both HPC and HPC 2.0 methods produce cells with consistently high expression of the primitive HPC marker CD43. (Top) Quantification of flow cytometry analysis from four independent iPSC lines per method ( $n=3$ wells/line) reveal a similarly high proportion of cells (> 90\%) that express the primitive HPC marker CD43 following differentiation with either our previous or currently described approach. In contrast, two other primitive HPC markers, CD41 and CD235a, exhibit relatively low and heterogeneous expression within this CD43+ population (Middle). Representative FACS plots demonstrating typical CD43+ histograms (pre gated for live, single cells). Gates were drawn based on FMO (fluorescence minus one) controls. (Bottom) Heatmap from RNA sequencing of iPS-HPC samples shows similar gene expression
\end{abstract} levels for CD43, CD41, and CD235a. (TIF $45670 \mathrm{~kb}$ )

Additional file 3: Table S1. Significantly changed genes between iPSmicroglia, iPS-microglia 2.0, CD14 monocytes, and CD16 monocytes. (XLSX $1562 \mathrm{~kb})$ 
Additional file 4: Table S3. Differential expression analysis of iPS-HPC versus iPS-HPC2.0. (XLSX $11 \mathrm{~kb}$ )

Additional file 5: Table S2. Differential expression analysis of iPSmicroglia versus iPS-microglia2.0. (XLSX $80 \mathrm{~kb}$ )

Additional file 6: Table S4. Differential expression analysis comparing IDE-treated microglia to TGFB control microglia or fetal and adult cultured microglia. (XLSX $11597 \mathrm{~kb}$ )

Additional file 7: Table S5. Catelog numbers for all materials used in this manuscript. (XLSX $45686 \mathrm{~kb}$ )

\section{Abbreviations}

AD: Alzheimer's disease; C1Q: Complement component 1q; CD: Cluster of differentiation; CR1: Complement receptor 1; CRISPR: Clustered regularly interspersed short palindromic repeats; CX3CL1: Fractalkine; CX3CR1: C-X3-C motif chemokine receptor 2; DPBS: Dulbecco's phosphate buffered saline; FACS: Fluorescence-associated cell-sorting; FDR: False discovery rate; GMCSF: Granulocyte-macrophage colony-stimulating factor; HC1: Histone H1like; HEXB: Hexosaminidase subunit beta; HPC: Hematopoietic progenitor cell; IBA1: Ionized calcium binding adaptor molecule 1; IDE: Inducer of definitive endoderm; IL2rg: Interleukin 2 receptor subunit gamma; IL-34: Interleukin 34; iPS/iPSCs: Induced pluripotent stem cells; MACS: Magnetic-associated cellsorting; MCSF/CSF1: Macrophage colony-stimulating factor; MITRG: Microglia transplant compatible mouse strain see https://www.jax.org/strain/017711; mRNA: messenger RNA; MS4A4A: Membrane-spanning 4-domains subfamily A member 4A; OLFML3: Olfactomedin like 3; P2RY12: Purinergic receptor P2Y12; PBS: Phosphate buffered saline; PC: Principal component; pHrodo: $\mathrm{pH}$ sensitive dyes; RABC3: Ras related protein Rab3c; Rag2: Recombination activating 2; RNA: Ribonucleic acid; TGF $\beta$ : Transforming growth factor $\beta$ TPO: Thrombopoietin; TREM2: Triggering receptor expressed on myeloid cells

\section{Acknowledgements}

The authors would like to thank Eric Martinez for his help with cell culture and RNA isolation The authors would like to thank the Genomics core at UC particularly Melanie Oakes, Christina Lin, and Seung-Ah Chung. We would also like to thank Dr. Jennifer Atwood of UCI's Institute for Immunology for her assistance with FACS. Finally, the authors would like to thank Amrita Choudhury from Genialis for her excellent assistance in RNAsequencing analysis.

\section{Funding}

This work was supported by NINDA T32 NS082174 (A.M), NIH AG048099, NIH AG056303 and CIRM RT3-07893 (M.B.J.) and a generous gift from the Susan Scott Foundation. iPSC lines were generated by the UCI-ADRC iPS cell core funded by NIH AG016573.

The UCl Genomics High Throughput Facility Shared Resource was supported by NIH grants P30CA-062203, 1S10RR025496-01, 1S100D010794-01, and 1S100D021718-01.

\section{Availability of data and materials}

The datasets generated and analyzed in this study are available through GEO Series accession number GSE117829, https://www.ncbi.nlm.nih.gov/geo/ query/acc.cgi?acc=GSE117829. The datasets generated though [11] and reanalyzed for this study are available through GEO Series accession number GSE89189, https://www.ncbi.nlm.nih.gov/geo/query/acc.cgi?acc=GSE89189.

\section{Authors' contributions}

$M B J$ and $A M$ designed experiments. MBJ, AM, and MC prepared the manuscript. CT generated iPSC cells and differentiated HPCs. AM differentiated HPCs and iPS-microglia for RNA sequencing, functional assays, and transplantation. $\mathrm{AM}$ and $\mathrm{JH}$ analyzed RNA-sequencing data. MC, $\mathrm{HD}$, and $\mathrm{JH}$ transplanted iPS-microglia and iPS-microglia 2.0, collected brains, and performed immunohistochemistry. MC and MBJ performed confocal microscopy. All authors read and approved the final manuscript.

\section{Authors' information}

Not applicable

\section{Ethics approval and consent to participate}

All experiments were carried out according to human stem cell (hSCRO) and animal use (IACUC) protocols that were approved by the University of California, Irvine.

\section{Consent for publication}

Not applicable.

\section{Competing interests}

The authors declare that they have no competing interests.

\section{Publisher's Note}

Springer Nature remains neutral with regard to jurisdictional claims in published maps and institutional affiliations.

\section{Author details}

${ }^{1}$ Department of Neurobiology \& Behavior, University of California, 3014 Gross Hall, 845 Health Science Rd, Irvine, CA 92697-4545, USA. ${ }^{2}$ Sue and Bill Gross Stem Cell Research Center, University of California, 3014 Gross Hall, 845 Health Science Rd, Irvine, CA 92697-4545, USA. ${ }^{3}$ Institute for Memory Impairments and Neurological Disorders, University of California, 3014 Gross Hall, 845 Health Science Rd, Irvine, CA 92697-4545, USA.

Received: 3 August 2018 Accepted: 28 November 2018

Published online: 22 December 2018

\section{References}

1. Greter M, et al. Stroma-derived interleukin-34 controls the development and maintenance of langerhans cells and the maintenance of microglia. Immunity. 2012;37(6):1050-60.

2. Butovsky $\mathrm{O}$, et al. Identification of a unique TGF- $\beta$-dependent molecular and functional signature in microglia. Nat Neurosci. 2014;17(1):131-43.

3. Yamasaki $R$, et al. Differential roles of microglia and monocytes in the inflamed central nervous system. J Exp Med. 2014;211(8):1533-49.

4. Heneka MT, et al. Neuroinflammation in Alzheimer's disease. Lancet Neurol. 2015:14(4):388-405.

5. Endo F, et al. Astrocyte-derived TGF- $\beta 1$ accelerates disease progression in ALS mice by interfering with the neuroprotective functions of microglia and T cells. Cell Rep. 2015;11(4):592-604.

6. Radford RA, Morsch M, Rayner SL, Cole NJ, Pountney DL, Chung RS. The established and emerging roles of astrocytes and microglia in amyotrophic lateral sclerosis and frontotemporal dementia. Front Cell Neurosci. 2015;9:414

7. Schapansky J, Nardozzi JD, LaVoie MJ. The complex relationships between microglia, alpha-synuclein, and LRRK2 in Parkinson's disease. Neuroscience. 2015:302:74-88.

8. Bennett $M L$, et al. New tools for studying microglia in the mouse and human CNS. Proc Natl Acad Sci U S A. 2016:113(12):E1738-46.

9. Ueda Y, Gullipalli D, Song W-C. Modeling complement-driven diseases in transgenic mice: values and limitations. Immunobiology. 2016;221(10):1080-90.

10. Durafourt BA, et al. Comparison of polarization properties of human adult microglia and blood-derived macrophages. Glia. 2012;60(5):717-27.

11. Abud EM, et al. iPSC-Derived Human Microglia-like Cells to Study Neurological Diseases. Neuron. 2017;94(2):278-293.e9.

12. Muffat J, et al. Efficient derivation of microglia-like cells from human pluripotent stem cells. Nat Med. 2016;22(11):1358-67.

13. Haenseler W, et al. A Highly Efficient Human Pluripotent Stem Cell Microglia Model Displays a Neuronal-Co-culture-Specific Expression Profile and Inflammatory Response. Stem Cell Rep. 2017:8(6):1727-1742, 06

14. K. Takata et al., "Induced-Pluripotent-Stem-Cell-Derived Primitive Macrophages Provide a Platform for Modeling Tissue-Resident Macrophage Differentiation and Function," Immunity, vol. 47, no. 1, pp. 183-198.e6, Jul. 2017.

15. Pandya $\mathrm{H}$, et al. Differentiation of human and murine induced pluripotent stem cells to microglia-like cells. Nat Neurosci. 2017;20(5):753-9.

16. Douvaras $P$, et al. Directed differentiation of human pluripotent stem cells to microglia. Stem Cell Rep. 2017:8(6):1516-24

17. Ditadi A, Sturgeon CM. Directed differentiation of definitive hemogenic endothelium and hematopoietic progenitors from human pluripotent stem cells. Methods San Diego Calif. 2016;101:65-72, 15.

18. Abutbul $S$, et al. TGF- $\beta$ signaling through SMAD2/3 induces the quiescent microglial phenotype within the CNS environment. Glia. 2012;60(7):1160-71. 
19. Chen S, Luo D, Streit WJ, Harrison JK. TGF-beta1 upregulates CX3CR1 expression and inhibits fractalkine-stimulated signaling in rat microglia. J Neuroimmunol. 2002;133(1-2):46-55.

20. Elmore MRP, et al. Colony-stimulating factor 1 receptor signaling is necessary for microglia viability, unmasking a microglia progenitor cell in the adult brain. Neuron. 2014;82(2):380-97.

21. Borowiak M, et al. Small molecules efficiently direct endodermal differentiation of mouse and human embryonic stem cells. Cell Stem Cell. 2009;4(4):348-58.

22. Friedman BA, et al. Diverse brain myeloid expression profiles reveal distinct microglial activation states and aspects of Alzheimer's disease not evident in mouse models. Cell Rep. 2018;22(3):832-47.

23. Gosselin D, et al. An environment-dependent transcriptional network specifies human microglia identity. Science. 2017;356(6344):23.

24. Kan AA, et al. Protein expression profiling of inflammatory mediators in human temporal lobe epilepsy reveals co-activation of multiple chemokines and cytokines. J Neuroinflammation. 2012;9:207.

25. Garofalo S, et al. Environmental stimuli shape microglial plasticity in glioma. eLife. 2017;6:29.

26. M. Olah et al., "A transcriptomic atlas of aged human microglia," Nat. Commun., vol. 9, no. 1, p. 539, 072018.

27. Burns $A$, Jacoby $R$, Luthert $P$, Levy R. Cause of death in Alzheimer's disease Age Ageing. 1990;19(5):341-4.

28. Kukull WA, et al. Causes of death associated with Alzheimer disease: variation by level of cognitive impairment before death. J Am Geriatr Soc. 1994;42(7):723-6.

29. Wang $H$, et al. Increased hypothalamic microglial activation after viralinduced pneumococcal lung infection is associated with excess serum amyloid a production. J Neuroinflammation. 2018;15(1):200.

30. Ji P, Schachtschneider KM, Schook LB, Walker FR, Johnson RW. Peripheral viral infection induced microglial sensome genes and enhanced microglial cell activity in the hippocampus of neonatal piglets. Brain Behav Immun. 2016:54:243-51.

31. "BBDuk Guide," DOE Joint Genome Institute. [Online]. Available: https://jgi. doe.gov/data-and-tools/bbtools/bb-tools-user-guide/bbduk-guide/. Accessed 8 May 2018.

32. Bray NL, Pimentel H, Melsted P, Pachter L. Near-optimal probabilistic RNAseq quantification. Nat Biotechnol. 2016;34(5):525-7.

33. Love Ml, Huber W, Anders S. Moderated estimation of fold change and dispersion for RNA-seq data with DESeq2. Genome Biol. 2014;15(12).

34. "Genialis." https://www.genialis.com.

Ready to submit your research? Choose BMC and benefit from:

- fast, convenient online submission

- thorough peer review by experienced researchers in your field

- rapid publication on acceptance

- support for research data, including large and complex data types

- gold Open Access which fosters wider collaboration and increased citations

- maximum visibility for your research: over $100 \mathrm{M}$ website views per year

At $\mathrm{BMC}$, research is always in progress.

Learn more biomedcentral.com/submissions 